\title{
An Artificial Intelligence-based Multimodality- Integration for Precise Prediction of Prostate Cancer Lymph Node Metastasis: A Retrospective Two-center Study
}

\author{
Ying Hou \\ the first affiliated hospital of Nanjing Medical University \\ Jie Bao \\ First Affiliated Hospital of Soochow University

\section{Yang Song} \\ East China Normal University \\ Mei-Ling Bao \\ the first affiliated hospiatl of Nanjing Medical University

\section{Ke-Wen Jiang} \\ the first affiliated hospital of Nanjing Medical University \\ Jing Zhang \\ the first affiliated hospital of Nanjing Medical University

\section{Guang Yang}

East China Normal University

\section{Chun-Hong Hu}

the first affiliated hospital of soochow university

Hai-Bin Shi

the first affiliated hospital of Nanjing Medical University

\section{Xi-Ming Wang}

the first affiliated hospital of Soochow University

Yu-Dong Zhang ( $\square$ njmu_zyd@163.com )

Jiangsu Province Hospital and Nanjing Medical University First Affiliated Hospital

\section{Research}

Keywords: pelvic lymph node metastasis, prostate cancer, radiomics, deep learning

Posted Date: September 24th, 2020

DOI: https://doi.org/10.21203/rs.3.rs-80704/v1 
License: (c) (i) This work is licensed under a Creative Commons Attribution 4.0 International License. Read Full License 


\section{Abstract}

\section{Background}

Accurate identification of pelvic lymph node metastasis (PLNM) in patients with prostate cancer (PCa) is crucial for determining appropriate treatment options. However, there is no clear consensus on the integration of clinicopathological and imaging findings available to predict PLNM. Therefore, we built a Prostate Cancer Risk (PRISK) tool using an artificial intelligence-based multimodality-integration to obtain a precisely informed decision about whether to perform extended pelvic lymph node dissection (ePLND).

Methods

PRISK provides a novel precise risk assessment tool to reduce unnecessary ePLNDs while controlling PLNM missing rate. It was developed in 280 patients and verified in 71 patients internally and in 50 patients externally by integrating a set of radiologists' interpretations, clinicopathological factors and newly refined imaging indicators from MR images with radiomics machine learning and deep learning algorithms. Its clinical applicability was compared with Briganti and Memorial Sloan Kettering Cancer Center (MSKCC) nomograms.

Results

PRISK yielded the best diagnostic performance with areas under the receiver operating characteristic curve (AUC) of 0.932 (95\% Cl, 0.895-0.958), 0.924 (95\% Cl, 0.837-0.974) and 0.758 (95\% Cl, 0.616-0.868) in the training/validation, internal test and external test cohorts. If the No. of ePLNDs missed for risk assessment is controlled at $<2 \%$, PRISK can provide both higher No. of ePLNDs spared (PRISK $59.6 \%$ vs MSKCC $44.9 \%$ vs Briganti $37.7 \%$ ) and lower No. of false-positives (PRISK $59.3 \%$ vs MSKCC $70.1 \%$ and Briganti $72.7 \%$ ) as compared with MSKCC and Briganti score. In follow-up, patients stratified by PRISK showed significantly different biochemical recurrence rate after surgery.

Conclusions

PRISK offers a noninvasive clinical biomarker to predict PLNM for patients with PCa. It shows improved accuracy of diagnosis support and reduced overtreatment burdens for patients with findings suggested of PCa.

\section{Background}

Prostate cancer $(\mathrm{PCa})$ is one of the most common malignancies and the second leading cause of cancerrelated mortality in western men ${ }^{1}$. The presence of pelvic lymph node metastasis (PLNM), with the incidence of up to $15 \%$ in a patient with newly diagnosed $\mathrm{PCa}$, is an important prognostic factor, indicating great risk for biochemical recurrence and distant metastases after curative treatment 2,3 . Therefore, accurate pretreatment identification of PLNM in patients with PCa would have a significant impact on clinical decision making, the planning of treatments and prediction of outcomes for patients ${ }^{4}$. 
Pelvic lymph node dissection (PLND) or extended PLND (ePLND) is generally recommended for high-risk PCa patients who are undergoing radical prostatectomy (RP) ${ }^{4}$. However, there is no ideal preoperative tool available to select candidates for PLND or ePLND due to unsatisfying sensitivity and application limitation ${ }^{5}$. In the last few decades, risk assessment tools such as Briganti score, Memorial Sloan Kettering Cancer Center (MSKCC) nomogram and Partin tables were proposed for the prediction of PLNM ${ }^{6-8}$, while showing moderate predictive accuracy on internal and external validations ${ }^{9-12}$. Multiparametric magnetic resonance imaging (mpMRI) has been widely used to preoperatively characterize PCa and determine the tumor and nodal stage ${ }^{13}$. Lymph node over $8 \mathrm{~mm}$ in short-axis dimension on $\mathrm{T}_{2}$-weighted imaging $\left(\mathrm{T}_{2} \mathrm{WI}\right)$ and high signal intensity on diffusion weighted imaging (DWI) is generally recognized as suspicious for malignancy. However, the performance of mpMRI for direct interpretation of PLNM is relatively poor, with a sensitivity of less than $60 \%{ }^{14}$. Moreover, interpretation of mpMRI requires the expertise of experienced radiologists; accordingly, there is inconsistency across readers of varying experience. Recently, measurable imaging parameters such as tumor size, apparent diffusion coefficient (ADC), dynamic contrast-enhanced-derived Ktrans, and imaging-based staging of PCa have been regarded as promising predictors of PLNM ${ }^{15,16}$. An ideal risk assessment tool by integrating clinical and imaging information would consistently improve the predictive performance for PLNM, but this is lacking. Several studies had attempted to develop models for the prediction of PLNM from imaging findings and demographic factors ${ }^{17-19}$. Others have carried out on the application of artificial intelligence (Al) such as machine learning or deep learning methods, yielding higher accuracy than the Briganti and MSKCC nomograms ${ }^{20}$. Transforming full imaging findings, especially these high-dimensional machine-learning radiomics features and/or deep learning-generative features, into a clinically interpretable signature is a potential alternative for improved prognostic and predictive accuracy in patients with localized PCa. However, there are challenges associated for such analyses. First, there are multi-modalities data generated in different clinical workflows and procedures; a clinical tool should leverage the integration and interactions of multi-modal features for risk-stratification analysis. Second, in the analysis of highresolution imaging data, machine learning or deep learning has shown promising performance, but requires more precisely guided modeling and confronts small-sample learning problems. Third, for largescale multi-modality data, the traditional analyzing has to do with its limitation in dealing with these highdimensional data. As every algorithm has their strengths and weaknesses; there is no single learning algorithm that works best for every problem.

To fill this gap, we described a Prostate Cancer Risk Calculator (PRISK) for PLNM assessment and to aid in PLND decision in PCa patients. PRISK incorporates a set of demographic/clinical factors and imaging indicators extracted by in-depth Al-based analysis. Our hypothesis is that the demographic/clinical factors combined with Al-based imaging biomarkers might provide better prediction and stratification of PLNM. Our study included 401 patients with PCa from two medical centers, consisting of 280 data sets for PRISK model development, and an independent set of 71 patients for internal test and 50 patients for external test. The findings of current study showed that our PRISK can 
identify subtle patterns accurately. It generates a more optimized risk score for the decision to PLND in surgical candidates.

\section{Methods}

\section{Ethical information and Study cohort}

This study was retrospective and approved by the local Institutional Review and need for written informed consent was waived. All included of consecutive patients who underwent prostate mpMRI at two tertiary care medical centers were reviewed. All procedures performed in studies involving human participants were in accordance with the 1964 Helsinki declaration and its later amendments. A total of 351 consecutive patients between Jan 2012 and Jun 2019 from the First Affiliated Hospital of Nanjing Medical University (NUH) and 50 PCa patients between January 2017 and December 2019 from the First Affiliated Hospital of Soochow University (SUH), who underwent both radical prostatectomy and ePLND, were identified and formed the primary cohort. A flow diagram of patient selection with inclusion and exclusion criteria is provided in Supplementary Fig. S1.

\section{Preoperative clinical characteristics and histological data}

Clinical and histopathologic data were obtained from the medical records. Preoperative clinical characteristics included the age, serum prostate-specific antigen (PSA), PSA density (PSAD), clinical Tstage ( $\leq T 1 c, T 2 a-c, \geq T 3$ ), biopsy Gleason score $(\leq 3+3,3+4,4+3$, and $\geq 4+4)$, percentage of positive cores, as well as perineural invasion (absent or present). The histologic parameters included Gleason score $(\leq 3+3,3+4,4+3$, and $\geq 4+4)$, surgical margin, extracapsular extension (ECE), seminal vesicle invasion (SVI) and PLNM (absent or present). Histopathologic results of ePLND including the total number of resected lymph nodes and total number of positive nodes were recorded.

Transrectal ultrasound (TRUS)-guided cognitive biopsy and/or targeted TRUS/MRI-fusion biopsy was performed followed by a standard 13-core systematic biopsy after mp-MRI scans. RP with ePLND was performed in high-risk patients classified by the EAU risk group classification criteria. All biopsies and surgical specimens were prepared and examined by two pathologists who had 10-yr experience in urologic pathology according to the ISUP 2005 recommendations.

\section{Follow-up}

The first postoperative visit was 6 weeks later after RP and ePLND and then patients were consistently followed-up at intervals of 3 to 6 months based on PSA. The time of biochemical recurrence (BCR) was recorded. Patients were censored in case of emigration, or on $30^{\text {th }} \mathrm{Jul} 2020$, whichever came first. The definition of BCR was referred to criteria previously reported 21,22 .

\section{Prostate mp-MRI examination and sequence preprocess}


MpMRI images were acquired prior to prostatic biopsy on two same 3.0-T MR scanner (Verio and Skyra; Siemens, Erlangen, Germany) at the two institutes with a pelvic phased-array coil, complaint with European Society of Uro-Radiology guidelines. The scan protocol is a combination of transverse T1weighted, transverse, coronal, and sagittal T2-weighted imaging, and transverse diffusion-weighted imaging sequences. The parameters are listed in Supplementary Table 1. Besides, the ADC value was measured by expending a mono-exponential fitting model.

The elastic transform from low-resolution DWI and ADC to high-resolution T2-w images was estimated by an Elastix software package (v. 4.10) referring to the suggested parameter file "par0001bspline16". The parameter configuration for the registration was set according to Klein et al's work ${ }^{23}$. The aligned DWI and $A D C$ had the same resolution, field of view and the orientation compared with $\mathrm{T}_{2} \mathrm{WI}$.

\section{Radiologists' interpretation}

The mpMRI exam were retrospectively interpreted based upon the guidelines of ESUR by two genitourinary radiologists at two institutions (Reader 1, 10-year experience; and Reader 2, 15-year experience in prostatic MRI in NUH; Reader 3, 5-year experience; and Reader 4, 10-year experience in prostatic MRI in SUH;) respectively, who were blinded to the histological results and all clinical information. In each patient, the radiologists identified the leading cancer lesion, referring to those with the higher Prostate Imaging and Reporting and Data System (PI-RADS) version 2.1 (v2.1) score or larger diameter if the score was the same, and the following imaging features were recorded: (i) prostate volume; (ii) zone of lesion origin (peripheral zone (PZ) or transitional zone(TZ)); (iii) shape of the lesion (regular or irregular); (iv) margin of the lesion (well-defined or ill-defined); (v) tumor max diameter; (vi) volumetric mean ADC value; (vii) PI-RADS score (PI-RADS 1-5) ${ }^{24,25}$; (viii) MRI T-stage ( $\leq$ T1c, T2a, T2b, $\geq$ T2c); (ix) MRI-based ECE, SVI, and PLNM (absent or present). The definition of MRI-based imaging features was described in Supplementary Data 1, referring to criteria previously reported ${ }^{20,26}$. All cases were interpreted individually first and then re-reviewed in tandem by the two readers 4 weeks after the individual evaluation. Individual readings were used for the calculation of inter-observer agreement, and the re-review interpretation of both readers was used for the following model constructions. Any disagreement at re-review was discussed until a final standard consensus was generated.

\section{Radiomics machine learning and deep learning}

PCa lesions were manually segmented by two genitourinary fellows (Reader 5 and Reader 6 ) independently. The entire volume of interest ( $\mathrm{VOI}$ ) of the tumor were drawn on the base of radiologichistologic correlation slice by slice. For the patient with multiple lesions, only the dominant lesion was segmented. All sequences and the corresponding VOIs were resampled to the inner-resolution of $0.5 \times 0.5$ $\mathrm{mm}^{2}$ by Bicubic methods.

Radiomics features including shapes (14), histograms (18), textures (75), and wavelets (774) were extracted from $\mathrm{T}_{2} \mathrm{Wl}, \mathrm{DWI}$ with $\mathrm{b}=1500 \mathrm{~s} / \mathrm{mm}^{2}$, and $\mathrm{ADC}$ by PyRadiomics 
(https://pyradiomics.readthedocs.io/en/latest/\#) ${ }^{27}$. Since the low resolution of the diffusion images, we did not extract wavelet-based features from DWI and ADC. Finally, a total of 2553 features were obtained. Each feature was normalized to a similar scale to avoid scale effects. The features matrix was normalized by Z-score normalization, which maps each feature with mean of zero and standard deviation of one. Pearson product-moment correlation coefficient was estimated between each pair of features and remove random one if the value was larger than 0.9 .

The entire-volumetric radiomics features focus on the inner regions of $\mathrm{PCa}$. We further investigated a tumor-related region around the PCa to extract deep learning-embedded features. The deep learningembedded features adopted a transfer learning technique based on a VGG-19 networks which is pretrained on ImageNet ${ }^{28}$. For each case, the axis-slice with largest area of the PCa cross section was selected from axial $T_{2} W I$, high-b DWI and ADC images. In order to obtain the representative imaging features of the target lesion, we used hand-cropped ROI as an attention to gate the VGG-19 model training (i.e., regions around the $\mathrm{PCa}$ ) in the center slice of the MRI scan. For the image embedding, we used the penultimate layer of model to produce a total of 12, 288 new feature vectors, serving as another set of deep learning(DL)-derived imaging features.

A random forests classifier, which combined the concepts of feature selection and step model training was used to build radiomics and DL signatures for predicting PLNM. Cross-validation was applied on the training cohort to optimize the hyper-parameter of each methods and one-standard error was used to determine the number of the features. In random forests algorithm, each tree is developed from a bootstrap sample from the training data. When developing individual trees, an arbitrary subset of attributes is drawn from which the best attribute for the split is selected. The final model is based on the majority vote from individually developed trees in the forest. Regarding feature selection, we assessed the features using a mean decrease Gini index (MDGI). The MDGI represents the importance of individual features for correctly classifying a residue into linker and non-linker regions. The MDGI was calculated by classifying randomly selected linker features and non-linker features, and the mean MDGI was calculated as the averaged MDGI over 100 trials. The mean MDGI z-score of each feature was calculated as: , where is the mean MDGI of the feature dedicated; and $\sigma$ is the standard deviation of all mean MDGI. Vector elements with MDGI Z-Score larger than 1.5 were selected as optimum feature candidates. The direct output values of random forests classifier do not show probabilities of PLNM positivity of PCa, so we converted their output values to probabilities $(P i)$ by applying a sigmoid function as follows: $P_{i}$ $=1 /\left(1+\exp \left(-x_{i}\right)\right)$, where $x_{i}$ is the classifier output value. The value of $P_{i}$ indicates the probability that the observation is PLNM (+). By this, the outputs of radiomics machine learning (RML) and VGG-19 models (designated RML score and DL score) were obtained, respectively.

\section{Development, Performance, and Validation of the Predictive Model}

Our primary aim is to develop an interpretability-enabled Al model for discriminating patients between with PLNM or not. We randomly split the data of NUH into training $(n=280)$ and test $(n=71)$ group, 
respectively, for model development and internal test. We also used the data from SUH with 50 patients for external test.

The flowcharts of imaging analysis and PRISK development are descripted in Fig. 1. The PRISK model is comprised of 20 multi-modalities data derived features including 7 clinicopathological variables such as age, PSA, PSAD, clinical T-stage, biopsy grade, percentage of positive cores and perineural invasion; and 11 radiological findings such as prostate volume, zone of lesion origin, shape, margin, tumor size, $A D C$, PI-RADS score, MRI T-stage, MRI-based ECE, SVI and PLNM; and two Al-derived imaging signatures such as RML score and DL score. The PRISK model was trained using an open-source AutoGluon framework (https://github.com/awslabs/autogluon) for auto Al analysis. Different to other auto Al frameworks, AutoGluon employs a novel form of multilayer stack ensembling. The first layer of AutoGluon has $n$ types of base learners including extremely randomized trees, k-nearest neighbors, gradient boosting machine, random forests and a tabular neural network, whose outputs are concatenated and then fed into the next layer, which itself consists of multiple stacker models. These stackers then act as base models to an additional layer. It merely employs random search for hyperparameter tuning, model selection, ensembling, feature engineering, data preprocessing, data splitting, etc., thus offered us to implement all strategies for hyperparameter tuning, feature selection, model selection and ensemble.

\section{Statistical analysis}

The detailed difference of clinicopathological factors, radiological parameters, RML and DL signatures of PLNM-absent and PLNM-present were compared by t-test or Mann-Whitney U test. The discrimination performance of predictive models was quantified by the area under the ROC curve (AUC) value in the primary training data and internally validated in the independent test data. Diagnostic sensitivity (SEN), specificity (SPE), accuracy (ACC) were calculated at a cutoff point that maximized the value of the Youden index. Additionally, true-positive and false-positive rates, weighted by the odds of the selected threshold probability of risk, were assessed to evaluate the clinical usefulness and net benefits of the developed PRISK model.

Disease-specific survival was computed from the date of surgery to date of death or censored at the date of last follow-up. Progression-free survival was defined as the interval between surgery and PSA detection of biochemical recurrence, last follow-up, or death. Survival curves were generated with the Kaplan-Meier method and compared by a two-sided log-rank tests. The statistical analysis was conducted with an $\mathrm{R}$ package (version 3.3.4; http://www.Rproject.org). The reported statistical significance levels were all twosided, with statistical significance set at 0.05 .

\section{Results}

\section{Baseline characteristics}

Out of all patients included, presence of histologic PLNM was diagnosed in explanted tissue of 70/401 patients (17.5\%), with 64/351(18.2\%) in NUH and 6/50 (12\%) in SUH. The demographic/clinical factors 
included the serum prostate-specific antigen (PSA), PSA density (PSAD), clinical T-stage, biopsy Gleason score, number of positive cores and perineural invasion. The radiographic features interpreted by dedicated radiologists included the prostate volume, zonal location of $\mathrm{PCa}$, tumor shape, tumor margin, tumor size, tumor volumetric ADC value, Prostate Imaging and Reporting and Data System (PI-RADS) score. MRI-based T-stages such as extracapsular extension (ECE), seminal vesicle invasion (SVI) and LN status were also described. Detailed baseline characters of the patients are summarized in Table 1. 
Table 1: The baseline characteristics of PCa patients between PLNM (-) and PLNM (+) in NUH and SUH

\begin{tabular}{|c|c|c|c|c|c|c|}
\hline & NUH & & & SUH & & \\
\hline Variable & $\begin{array}{l}\text { PLNM (-) } \\
\mathrm{n}=\mathbf{2 8 7}\end{array}$ & $\begin{array}{l}\text { PLNM } \\
\begin{array}{l}(+) \\
n=64\end{array}\end{array}$ & $p$ & $\begin{array}{l}\text { PLNM }(-) \\
\mathrm{n}=\mathbf{4 4}\end{array}$ & $\begin{array}{l}\text { PLNM (+) } \\
\mathrm{n}=6\end{array}$ & $p$ \\
\hline $\operatorname{Age}(\mathrm{y})$, mean $(\mathrm{std})$ & $69.5(6.6)$ & $\begin{array}{l}67.5 \\
(7.2)\end{array}$ & $0.032 \dagger$ & $70.1(6.3)$ & $66.2(7.4)$ & $0.160+$ \\
\hline $\begin{array}{l}\mathrm{PSA}(\mathrm{ng} / \mathrm{mL}) \text {, mean } \\
\text { (range) }\end{array}$ & $\begin{array}{l}29.9(1.4- \\
591.0)\end{array}$ & $\begin{array}{l}75.6 \\
(3.9- \\
676.0)\end{array}$ & $0.000 \dagger$ & $\begin{array}{l}31.2(3.3- \\
100.0)\end{array}$ & $\begin{array}{l}77.6 \\
(22.6- \\
200.1)\end{array}$ & $0.155+$ \\
\hline $\begin{array}{l}\mathrm{PSAD}(\mathrm{ng} / \mathrm{mL} / \mathrm{cc}) \text {, } \\
\text { mean (range) }\end{array}$ & $0.8(0.1-9.2)$ & $\begin{array}{l}1.6(0.1- \\
16.3)\end{array}$ & $0.008+$ & $0.8(0.1-3.3)$ & $\begin{array}{l}1.8(0.5- \\
5.3)\end{array}$ & $0.008+$ \\
\hline Clinical T-stage & & & $0.031^{\star}$ & & & $0.328 *$ \\
\hline T1c or less & $\begin{array}{l}180 / 287 \\
(62.7)\end{array}$ & $\begin{array}{l}32 / 64 \\
(50.0)\end{array}$ & & $\begin{array}{l}29 / 44 \\
(65.9)\end{array}$ & $3 / 6(50.0)$ & \\
\hline T2a-c & $86 / 287(30.0)$ & $\begin{array}{l}30 / 64 \\
(46.9)\end{array}$ & & $\begin{array}{l}13 / 44 \\
(29.5)\end{array}$ & 2/6 (33.3) & \\
\hline T3 or more & $21 / 287(7.3$ & $\begin{array}{l}2 / 64 \\
(3.1)\end{array}$ & & $2 / 44(4.5)$ & $1 / 6(16.7)$ & \\
\hline $\begin{array}{l}\text { Prostate volume } \\
\left(\mathrm{cm}^{3}\right) \text {, mean (range) }\end{array}$ & $\begin{array}{l}39.0(11.2- \\
189.6)\end{array}$ & $\begin{array}{l}50.4 \\
(11.2- \\
117.7)\end{array}$ & $0.000 \dagger$ & $\begin{array}{l}43.8(15.7- \\
111.1)\end{array}$ & $\begin{array}{l}46.5(36.4- \\
61.0)\end{array}$ & $0.753+$ \\
\hline Tumor shape & & & $0.000 *$ & & & $0.650 *$ \\
\hline Regular & 138/287(48.1) & $\begin{array}{l}6 / 64 \\
(9.4)\end{array}$ & & $\begin{array}{l}16 / 44 \\
(36.4)\end{array}$ & $1 / 6(16.7)$ & \\
\hline Irregular & $\begin{array}{l}149 / 287 \\
(51.9)\end{array}$ & $\begin{array}{l}58 / 64 \\
(90.6)\end{array}$ & & $\begin{array}{l}28 / 44 \\
(63.6)\end{array}$ & $5 / 6(83.3)$ & \\
\hline Zone of tumor origin & & & $0.048^{\star}$ & & & $1.000 *$ \\
\hline TZ & $91 / 287(31.7)$ & $\begin{array}{l}12 / 64 \\
(18.8)\end{array}$ & & $11 / 44(25.0)$ & $1 / 6(16.7)$ & \\
\hline $\mathrm{PZ}$ & $\begin{array}{l}196 / 287 \\
(68.3)\end{array}$ & $\begin{array}{l}52 / 64 \\
(81.2)\end{array}$ & & $\begin{array}{l}33 / 44 \\
(75.0)\end{array}$ & $5 / 6(83.3)$ & \\
\hline Tumor margin & & & $0.000 *$ & & & $1.000 *$ \\
\hline Well-defined, n (\%) & $\begin{array}{l}126 / 287 \\
(43.9)\end{array}$ & $\begin{array}{l}5 / 64 \\
(7.8)\end{array}$ & & 8/44 (18.2) & $1 / 6(16.7)$ & \\
\hline III-defined, n (\%) & $\begin{array}{l}161 / 287 \\
(56.1)\end{array}$ & $\begin{array}{l}59 / 64 \\
(92.2)\end{array}$ & & $\begin{array}{l}36 / 44 \\
(81.8)\end{array}$ & $5 / 6(83.3)$ & \\
\hline
\end{tabular}




\begin{tabular}{|c|c|c|c|c|c|c|}
\hline $\begin{array}{l}\text { Tumor max diameter } \\
\text { (cm), mean (range) }\end{array}$ & $1.7(0.4-5.9)$ & $\begin{array}{l}3.1(0.7- \\
6.3)\end{array}$ & $0.001 \dagger$ & $2.1(0.5-5.4)$ & $\begin{array}{l}3.1(1.4- \\
4.1)\end{array}$ & $0.055 t$ \\
\hline $\begin{array}{l}\text { Mean ADC }\left(\times 10^{-3}\right. \\
\left.\mathrm{s} / \mathrm{mm}^{2}\right) \text {, mean }(\mathrm{std})\end{array}$ & $0.8(0.2)$ & $0.8(0.2)$ & $0.042 \dagger$ & $1.0(0.2)$ & $0.9(0.1)$ & $0.829+$ \\
\hline PI-RADS score & & & $0.000^{\star}$ & & & $1.000 *$ \\
\hline $1-2$ & $19 / 287(6.6)$ & $\begin{array}{l}0 / 64 \\
(0.0)\end{array}$ & & $3 / 44$ (6.8) & $0 / 6(0.0)$ & \\
\hline 3 & 40/287 (13.9) & $\begin{array}{l}0 / 64 \\
(0.0)\end{array}$ & & $2 / 44(4.5)$ & $0 / 6(0.0)$ & \\
\hline 4 & 95/287 (33.1) & $\begin{array}{l}4 / 64 \\
(6.3)\end{array}$ & & 8/44 (18.2) & $1 / 6(16.7)$ & \\
\hline 5 & $\begin{array}{l}133 / 287 \\
(46.3)\end{array}$ & $\begin{array}{l}60 / 64 \\
(93.7)\end{array}$ & & $\begin{array}{l}31 / 44 \\
(70.5)\end{array}$ & $5 / 6(83.3)$ & \\
\hline MRI-based stage & & & $0.000^{\star}$ & & & $1.000 *$ \\
\hline T1c or less & $42 / 287(14.6)$ & $\begin{array}{l}0 / 64 \\
(0.0)\end{array}$ & & $2 / 44(4.5)$ & $0 / 6(0.0)$ & \\
\hline T2a & $98 / 287(34.1)$ & $\begin{array}{l}2 / 64 \\
(3.1)\end{array}$ & & $\begin{array}{l}14 / 44 \\
(31.8)\end{array}$ & $2 / 6(33.3)$ & \\
\hline $\mathrm{T} 2 \mathrm{~b}$ & $24 / 287(8.4)$ & $\begin{array}{l}5 / 64 \\
(7.8)\end{array}$ & & 4/44 (9.1) & $0 / 6(0.0)$ & \\
\hline T2c or more & $\begin{array}{l}123 / 287 \\
(42.8)\end{array}$ & $\begin{array}{l}57 / 64 \\
(89.1)\end{array}$ & & $\begin{array}{l}24 / 44 \\
(54.5)\end{array}$ & $4 / 6(66.7)$ & \\
\hline MRI-ECE+ & $90 / 287$ (31.4) & $\begin{array}{l}55 / 64 \\
(85.9)\end{array}$ & $0.000^{*}$ & $\begin{array}{l}15 / 44 \\
(34.1)\end{array}$ & $3 / 6(50.0)$ & $0.654^{*}$ \\
\hline MRI-SVI+ & 29/287 (10.1) & $\begin{array}{l}43 / 64 \\
(67.2)\end{array}$ & $0.000^{\star}$ & 9/44 (20.5) & $2 / 6$ (33.3) & $0.601^{*}$ \\
\hline MRI-LNI+ & $15 / 287(5.2)$ & $\begin{array}{l}36 / 64 \\
(56.3)\end{array}$ & $0.000^{*}$ & $\begin{array}{l}10 / 44 \\
(22.7)\end{array}$ & $2 / 6(33.3)$ & $0.621^{*}$ \\
\hline Biopsy findings & & & $0.000^{\star}$ & & & $0.504^{*}$ \\
\hline GS $3+3$ & $70 / 287$ (24.4) & $\begin{array}{l}3 / 64 \\
(4.7)\end{array}$ & & $0 / 44(0.0)$ & $0 / 6(0.0)$ & \\
\hline GS $3+4$ & $63 / 287(22.0)$ & $\begin{array}{l}5 / 64 \\
(7.8)\end{array}$ & & 9/44 (20.5) & $0 / 6(0.0)$ & \\
\hline GS 4+3 & $77 / 287$ (26.8) & $\begin{array}{l}21 / 64 \\
(32.8)\end{array}$ & & $\begin{array}{l}11 / 44 \\
(25.0)\end{array}$ & $1 / 6(16.7)$ & \\
\hline$G S \geq 4+4$ & $77 / 287(26.8)$ & $\begin{array}{l}35 / 64 \\
(54.7)\end{array}$ & & $\begin{array}{l}24 / 44 \\
(54.5)\end{array}$ & $5 / 6(83.3)$ & \\
\hline Percentage of & $0.4(0.0-1.0)$ & $0.7(0.2-$ & $0.000 \dagger$ & $0.5(0.1-1.0)$ & 0.8 (0.6- & $0.008+$ \\
\hline
\end{tabular}


positive cores,

median (range)

\section{Surgical findings}

\begin{tabular}{lllll}
\hline GS 3+3 & $33 / 287(11.5)$ & $\begin{array}{l}0 / 64 \\
(0.0)\end{array}$ & $0 / 44(0.0)$ & $0 / 6(0.0)$ \\
\hline GS 3+4 & $87 / 287(30.3)$ & $\begin{array}{l}4 / 64 \\
(6.3)\end{array}$ & $8 / 44(18.2)$ & $0 / 6(0.0)$ \\
\hline GS 4+3 & $97 / 287(33.8)$ & $\begin{array}{l}17 / 64 \\
(26.6)\end{array}$ & $\begin{array}{l}17 / 44 \\
(38.6)\end{array}$ & $0 / 6(0.0)$ \\
\hline GS $\geq 4+4$ & $71 / 287(24.7)$ & $\begin{array}{l}43 / 64 \\
(67.2)\end{array}$ & $\begin{array}{l}19 / 44 \\
(53.2)\end{array}$ & $6 / 6$ \\
\hline ECE+ & $76 / 287(33.0)$ & $\begin{array}{l}44 / 64 \\
(68.8)\end{array}$ & $13 / 44$ & $4 / 6(66.7)$ \\
\hline SVI+ & $31 / 287(13.5)$ & $\begin{array}{l}43 / 64 \\
(67.2)\end{array}$ & $5 / 44(11.4)$ & $2 / 6(33.3)$ \\
\hline SM+ & $91 / 287(39.6)$ & $\begin{array}{l}48 / 64 \\
(75.0)\end{array}$ & $\begin{array}{l}15 / 44 \\
(34.1)\end{array}$ & $4 / 6(66.7)$ \\
\hline LNI+ & $0 / 287(0.0)$ & $\begin{array}{l}64 / 64 \\
(100.0)\end{array}$ & $0 / 44(0.0)$ & $6 / 6$ \\
& & 798 & 238 & 71 \\
\hline $\begin{array}{l}\text { No. of nodes } \\
\text { dissected }\end{array}$ & 2758 & 248 & 0 & 19
\end{tabular}

Note. -Unless indicated otherwise, data are number of tumors, with percentages in parentheses. $\mathrm{NUH}=$ the First Affiliated Hospital of Nanjing Medical University. SUH = the First Affiliated Hospital of Soochow University. PSA = prostate serum antigen. PSAD = prostate serum antigen density. PI-RADS= Prostate Imaging and Reporting and Data System version 2.1[ref.37.38] ECE = extracapsular extension $. S V I=$ seminal vesicle invasion. $\mathrm{PLNM}=$ pelvic lymph node metastasis. $. \mathrm{S}=$ = Gleason Score . $\mathrm{RP}=$ radical prostatectomy. $\mathrm{SM}=$ surgical margin. IIndependent sample t test. * Chi-Square test.

\section{PRISK development}

The dynamic performance tuning of two types of imaging-signature models (RML score and DL score), and ensemble of the best-performing PRISK models are descripted in Fig. 2. The novelty of our model development is that the PRISK is built from an in-depth multimodality features/models ensembling using 10 dedicated Al-based learning algorithms, where the modalities and dedicated base models can be interpretable. Additionally, RML score, MRI-interpreted SVI and DL score were identified as three topranked predictors of PLNM; and model using stacking ensemble outperforms other base models in terms of importance score and AUC value. 
The resulting PRISK based on the model ensembling demonstrated good accuracy in estimating the risk of PLNM, with an area under the receiver operating characteristic curve (AUC) of $0.932(95 \% \mathrm{Cl}, 0.895$ $0.958)$ in training group, $0.924(95 \% \mathrm{Cl}, 0.837-0.974)$ in internal and $0.758(95 \% \mathrm{Cl}, 0.616-0.868)$ in external test group, respectively(Fig. 3a). The obtained PRISK score showed significant difference $(p<$ 0.001) between PLNM-absent and PLNM-present groups, and using an optimal threshold (PRISK score > 0.131 ) that maximizes the Youden index of the ROC analysis from the training/validation data, the PRISK model resulted in a sensitivity of $92.2 \%, 84.6 \%$ and $50.0 \%$ with a specificity of $81.2 \%, 84.5 \%$ and $84.0 \%$ in predicting PLNM in training, internal and external test sets, respectively(Fig. $\mathbf{3 b}$ ). In order to evaluate the integrated effects of different modalities/features, we compared the performance of PRISK with that of model using clinical factors (Clinical), clinical and ML-based radiomics factors (Clinical $+\mathrm{RML})$, clinical and DL-derived features (Clinical $+D L$ ), where the input to the model was a single-modality or fuse multimodalities. The PRISK demonstrated better performance than that of RML and DL, Clinical model, Clinical + RML model, and Clinical + DL model, respectively, suggesting interactive effects of those modality features (Fig. 3c).

\section{Clinical application}

As part of this study, we considered clinical implications of using PRISK assessment as a triage test to identify who are candidates for ePLND or who should be spared. We compared the true/false positive rates of PRISK to an established MSKCC and Briganti score for stratifying PLNM risk. As shown in Fig. 4, for internal-tested data, PRISK resulted in similar true positive rates while achieved notably lower false positive rates at threshold probabilities of PLNM $<60 \%$ compared to MSKCC and Briganti score. For external-tested data, our PRISK had lower true positive rates and lower false positive rates compared to MSKCC and Briganti score. However, according to the European Association of Urology (EAU) and National Comprehensive Cancer Network (NCCN) guideline for the treatment of PCa, if the No. of ePLNDs missed for risk assessment is controlled at $<2 \%$ (PRISK, $1.7 \%$ vs MSKCC $2.2 \%$ and Briganti $1.9 \%$ ), our PRISK can provide both higher No. of ePLNDs spared (PRISK: 239/401 [59.6\%] vs MSKCC: 180/401 [44.9\%] vs Briganti 151/401[37.7\%]) and lower No. of false-positives (PRISK, 96/162 [59.3\%] vs MSKCC, 155/221 [70.1\%] and Briganti, 178/245 [72.7\%]) as compared with MSKCC and Briganti score.

\section{Prognostic aspects of PRISK for biochemical recurrence:}

As of $30^{\text {th }} \mathrm{Jul} 2020$, we collected 331/401 (82.5\%) patients who had completed 2-yr BCR follow-up after surgery. The overall recurrence rate was $34.7 \%$ (115/331), with $36.4 \%(104 / 286)$ in NUH and $24.4 \%$ $(11 / 45)$ in SUH. The median BCR-free survival of the patients was 5.8 (range, 1.4-40.7) months, and in particular 5.2 (1.4-40.7) months for those with PLNM and 6.2 (1.4-38.1) months for those without PLNM (log-rank test, $p<0.001)$. Similar results were observed in the PRISK model: The median PFS was 4.3 (1.428.8) months for patients with PRISK-predicted PLNM presence and 8.0 (1.4-40.7) months for those with PRISK-predicted PLNM absence (log-rank test, $p<0.001$ ) (Fig. 5).

\section{Discussion}


The EAU and NCCN clinical guidelines recommend the use of Briganti and MSKCC nomograms based on clinical factors to estimate the risk of PLNM ${ }^{4,29}$. However, these tools are associated with moderate performance characteristics for not taking intraprostatic heterogeneity into consideration. With wide application of mpMRI in prostate, evaluation of PLNM on the basis of such morphologic criteria not only is unable to detect microscopic metastasis, but also leads to false-positive results, with the sensitivity of $10-96 \%$ and the specificity of $89-99 \%{ }^{14}$.

In this study, we built and validated a clinically interpretable PRISK calculator, where multimodalities data/features from clinical, biopsy and high-dimensional imaging data were incorporated using a novel auto Al-based framework. Our model has several novel elements compared with previously published nomograms. First, to our knowledge, this artificial intelligent-based risk assessment tool is the first for the prediction of nodal status by the in-depth integration of multimodality data/features in a routine clinical workflow. In this light, the assumption that imaging features obtained by in-depth Al-based analysis have the potential to discover imaging phenotypes of PCa that fails to be recognized visually by human experts, thus for improved prognostic and predictive performance. This deep multimodal interaction and data mining would provide a more extensive understanding of heterogeneity of PCa. Second, the study cohort consisted of a relatively large population and the PRISK was developed and tested internally and externally. Third, different from most of the studies focusing on the task of combined algorithm selection and hyperparameter optimization, where only the algorithm with the best performance was selected ${ }^{30,31}$, we applied an open-source auto Al-based analysis framework using stacked ensemble learning, that combining the aggregated predictions of the base models as its features and exploiting interactions between base models that offer enhanced predictive power.

Distinct facets of our results deserve attention. First, our results show that the newly built imaging hallmarks, i.e., RML score and DL score, are workable for the nodal staging. In the previous studies, attempts to adding radiological evaluations to clinical nomograms have shown promising results, with the AUC of $0.89-0.9516,20,32$. In current work, when the new imaging hallmarks were integrated with clinical factors simultaneously, the predictive performance of PRISK was significantly higher than that of any other method. Additionally, the two new imaging hallmarks, were superior to most of those clinical factors in the weighted ensemble model, suggesting their incremental role on PCa risk assessment. Our findings are consistent with those of previous studies, which used the similar computational approaches for breast cancer assessment ${ }^{33,34}$.

Second, we made head-to-head comparison of the performance of PRISK with MSKCC and Briganti nomogram. Patients with the risk threshold higher than $5 \%$ estimated by the MSKCC or Briganti nomogram are the candidates for ePLND on the EAU guideline ${ }^{29,35}$. The adoption of $5 \%$ threshold at Briganti nomogram in original cohort resulted in $65 \%$ ePLNDs spared at cost of $1.5 \%$ PLNM missed ${ }^{6}$. On the following external validation, using a $7 \%$ cutoff, the Briganti nomogram had an AUC of 0.79 and resulted in $56-70 \%$ ePLNDs spared at cost of $1.5-2.6 \%$ PLNM missed ${ }^{36-38}$. In our cohort, with a PLNM missing rate controlled less than $2 \%$, the PRISK resulted in higher no. of ePLNDs spared $(59.6 \%$ vs $44.9 \%$ 
vs $37.7 \%$ ) at lower cost of no. of PLNMs missed (1.7\% vs $2.2 \%$ vs $1.9 \%)$ than MSKCC and Briganti nomogram. Therefore, our PRISK, using an optimal threshold $8 \%$, can resulted in higher no. of ePLNDs spared at cost of lower PLNMs missed. Our preliminary results also showcase the PRISK even revealed a potential role in predicting the prognostic of disease progression risk preoperatively. We found that $\mathrm{PCa}$ patients after surgery reflected significantly different BCR-free survival in the subgroups stratified by PRISK, MSKCC and Briganti score, implying the prognostic relevance of our PRISK assessment on short and long-term management of patients. After all, this was a preliminary result in a small population, the prognostic aspects of PRISK on external independent cohorts warrant further validations.

However, as showed results in our external-tested cohort, the PRISK might not be able to completely replace currently available models such as MSKCC or Briganti nomogram, the accuracy of which have been internally and externally validated. The performance in the external set is inferior to the training/validation and internal test sets. This may be caused by the small test cohort and the distribution difference that the positive rate in the external validation cohort in relatively lower than training/validation and internal test cohorts. Besides, subjective radiologist-based interpretations lack of standardization may be another important factor exerting an influence on external validation. it also requires the expertise of experienced radiologists and there is inconsistency across readers of varying experience. Therefore, it is further required to test the generalization of PRISK in more external cohorts.

Our study had several limitations. First, because of its retrospective character, there were a large number of patients who did not undergo ePLND excluded, selection bias and treatment procedure might make influence. Second, the study has tested externally on a relatively small group of patients, and the validation on a larger scale of patients in multicenter is needed. Third, prospective application of an automatic decision support tool in a clinical scenario for PCa risk assessment involves a series of challenges such as individual variations in the pathological manifestations of disease and technical variations in imaging device, parameters and image processing. Therefore, in current stage, our PRISK might not be routinely available.

\section{Conclusion}

Therefore, we proposed a PRISK assessment tool for the prediction of PLNM in PCa patients using Albased multimodality integration and multi-algorithm ensembling. The interpretability of PRISK is particularly imperative towards building trustable Al-based tools for clinical applications. Adoption of this risk assessment tool allows to spare $59.6 \%$ of ePLND procedures at the cost of missing only $1.7 \%$ of PLNM cases, better than the current MKSCC and Briganti nomogram. Therefore, integrating pre-treatment factors with in-depth Al approach would provide us a great alternative to improve our routine procedure in patient management.

\section{Abbreviations}


prostate cancer (PCa); pelvic lymph node metastasis (PLNM); pelvic lymph node dissection (PLND); radical prostatectomy (RP); Memorial Sloan Kettering Cancer Center (MSKCC); multiparametric magnetic resonance imaging (mpMRI); diffusion weighted imaging (DWI); apparent diffusion coefficient (ADC); artificial intelligence (Al); Prostate Cancer Risk Calculator (PRISK); prostate-specific antigen (PSA); extracapsular extension (ECE); seminal vesicle invasion (SVI); Prostate Imaging and Reporting and Data System (PI-RADS); peripheral zone (PZ); transitional zone(TZ); volume of interest (VOI); the area under the ROC curve (AUC); sensitivity (SEN), specificity (SPE), accuracy (ACC); biochemical recurrence (BCR); radiomics machine learning ( $R M L)$; deep learning $(D L)$.

\section{Declarations}

\section{Ethics approval and consent to participate}

This study was retrospective and approved by the local Institutional Review (2016-SRFA-093) and need for written informed consent was waived.

\section{Consent for publication}

Not applicable

\section{Availability of data and materials}

All data generated or analysed during this study are included in this published article and its supplementary materials.

\section{Competing interests}

The authors declare that they have no competing interests.

\section{Funding}

Contract grant sponsor: Key research and development program of Jiangsu Province; contract grant number: BE2017756 (to Y.D.Z.); Contract grant sponsor: Suzhou Science and Technology Bureau-Science and Technology Demonstration Project; contract grant number: SS201808 (to X.M.W)

\section{Authors' contributions}

X.W. and Y.Z. conceived, designed and supervised the project; Y.H., J.B. M.B., K.J., C.H. and H.S. collected and pre-processed all data and performed the research; Y.H., and J.B. performed imaging data annotation and clinical data review; Y.S. and G.Y. proposed the model; Y.H. and J.B. drafted the paper; X.W. and Y.Z. revised the paper. All authors reviewed and approved the final version of article.

\section{Acknowledgements}

Not applicable 


\section{References}

[1] Siegel RL, Miller KD, Jemal A. Cancer statistics, 2018. 2018;68:7-30.

[2] Wilczak W, Wittmer C, Clauditz T, Minner S, Steurer S, Buscheck F, et al. Marked Prognostic Impact of Minimal Lymphatic Tumor Spread in Prostate Cancer. European urology. 2018;74:376-86.

[3] von Bodman C, Godoy G, Chade DC, Cronin A, Tafe LJ, Fine SW, et al. Predicting biochemical recurrence-free survival for patients with positive pelvic lymph nodes at radical prostatectomy. The Journal of urology. 2010;184:143-8.

[4] Mottet N, Bellmunt J, Bolla M, Briers E, Cumberbatch MG, De Santis M, et al. EAU-ESTRO-SIOG Guidelines on Prostate Cancer. Part 1: Screening, Diagnosis, and Local Treatment with Curative Intent. European urology. 2017;71:618-29.

[5] Muteganya R, Goldman S, Aoun F, Roumeguere T, Albisinni S. Current Imaging Techniques for Lymph Node Staging in Prostate Cancer: A Review. Frontiers in surgery. 2018;5:74.

[6] Briganti A, Larcher A, Abdollah F, Capitanio U, Gallina A, Suardi N, et al. Updated nomogram predicting lymph node invasion in patients with prostate cancer undergoing extended pelvic lymph node dissection: the essential importance of percentage of positive cores. European urology. 2012;61:480-7.

[7] Memorial Sloan Kettering Cancer Center. Dynamic prostate cancer nomogram: coefficients.

[8] Tosoian JJ, Chappidi M, Feng Z, Humphreys EB, Han M, Pavlovich CP, et al. Prediction of pathological stage based on clinical stage, serum prostate-specific antigen, and biopsy Gleason score: Partin Tables in the contemporary era. BJU international. 2017;119:676-83.

[9] Hueting TA, Cornel EB, Somford DM, Jansen H, van Basten JA, Pleijhuis RG, et al. External Validation of Models Predicting the Probability of Lymph Node Involvement in Prostate Cancer Patients. European urology oncology. 2018;1:411-7.

[10] Grivas N, Wit E, Tillier C, van Muilekom E, Pos F, Winter A, et al. Validation and head-to-head comparison of three nomograms predicting probability of lymph node invasion of prostate cancer in patients undergoing extended and/or sentinel lymph node dissection. European journal of nuclear medicine and molecular imaging. 2017;44:2213-26.

[11] Nason GJ, O'Connor EM, MacMahon D, Moss B, Considine SW, Cahill A, et al. Comparison of nomograms predicting lymph node invasion in patients undergoing radical prostatectomy for prostate cancer. Irish journal of medical science. 2018;187:33-7.

[12] Cimino S, Reale G, Castelli T, Favilla V, Giardina R, Russo GI, et al. Comparison between Briganti, Partin and MSKCC tools in predicting positive lymph nodes in prostate cancer: a systematic review and meta-analysis. Scandinavian journal of urology. 2017;51:345-50. 
[13] Yakar D, Debats OA, Bomers JG, Schouten MG, Vos PC, van Lin E, et al. Predictive value of MRI in the localization, staging, volume estimation, assessment of aggressiveness, and guidance of radiotherapy and biopsies in prostate cancer. Journal of magnetic resonance imaging : JMRI. 2012;35:20-31.

[14] Woo S, Suh CH, Kim SY, Cho JY, Kim SH. The Diagnostic Performance of MRI for Detection of Lymph Node Metastasis in Bladder and Prostate Cancer: An Updated Systematic Review and Diagnostic MetaAnalysis. AJR American journal of roentgenology. 2018;210:W95-w109.

[15] Porpiglia F, Manfredi M, Mele F, Bertolo R. Indication to pelvic lymph nodes dissection for prostate cancer: the role of multiparametric magnetic resonance imaging when the risk of lymph nodes invasion according to Briganti updated nomogram is $<5$. 2018;21:85-91.

[16] Wang L, Hricak H, Kattan MW, Schwartz LH, Eberhardt SC, Chen HN, et al. Combined endorectal and phased-array MRI in the prediction of pelvic lymph node metastasis in prostate cancer. AJR American journal of roentgenology. 2006;186:743-8.

[17] Rayn KN, Bloom JB, Gold SA, Hale GR, Baiocco JA, Mehralivand S, et al. Added Value of Multiparametric Magnetic Resonance Imaging to Clinical Nomograms for Predicting Adverse Pathology in Prostate Cancer. The Journal of urology. 2018;200:1041-7.

[18] Alberts AR, Roobol MJ, Verbeek JFM, Schoots IG, Chiu PK, Osses DF, et al. Prediction of High-grade Prostate Cancer Following Multiparametric Magnetic Resonance Imaging: Improving the Rotterdam European Randomized Study of Screening for Prostate Cancer Risk Calculators. European urology. 2019;75:310-8.

[19] Morlacco A, Sharma V, Viers BR, Rangel LJ, Carlson RE, Froemming AT, et al. The Incremental Role of Magnetic Resonance Imaging for Prostate Cancer Staging before Radical Prostatectomy. European urology. 2017;71:701-4.

[20] Hou Y, Bao ML, Wu CJ, Zhang J, Zhang YD. A machine learning-assisted decision-support model to better identify patients with prostate cancer requiring an extended pelvic lymph node dissection. 2019;124:972-83.

[21] Cookson MS, Aus G, Burnett AL, Canby-Hagino ED, D'Amico AV, Dmochowski RR, et al. Variation in the definition of biochemical recurrence in patients treated for localized prostate cancer: the American Urological Association Prostate Guidelines for Localized Prostate Cancer Update Panel report and recommendations for a standard in the reporting of surgical outcomes. The Journal of urology. 2007;177:540-5.

[22] Brockman JA, Alanee S, Vickers AJ, Scardino PT, Wood DP, Kibel AS, et al. Nomogram Predicting Prostate Cancer-specific Mortality for Men with Biochemical Recurrence After Radical Prostatectomy. European urology. 2015;67:1160-7. 
[23] Klein S, Staring M Fau - Murphy K, Murphy K Fau - Viergever MA, Viergever Ma Fau - Pluim JPW, Pluim JP. elastix: a toolbox for intensity-based medical image registration.

[24] Weinreb JC, Barentsz JO, Choyke PL, Cornud F, Haider MA, Macura KJ, et al. PI-RADS Prostate Imaging - Reporting and Data System: 2015, Version 2. European urology. 2016;69:16-40.

[25] Turkbey B, Rosenkrantz AB, Haider MA, Padhani AR, Villeirs G, Macura KJ, et al. Prostate Imaging Reporting and Data System Version 2.1: 2019 Update of Prostate Imaging Reporting and Data System Version 2. European urology. 2019;76:340-51.

[26] Zhang YD, Wu CJ, Bao ML, Li H, Wang XN, Liu XS, et al. MR-based prognostic nomogram for prostate cancer after radical prostatectomy. Journal of magnetic resonance imaging : JMRI. 2017;45:586-96.

[27] van Griethuysen JJM, Fedorov A, Parmar C, Hosny A, Aucoin N, Narayan V, et al. Computational Radiomics System to Decode the Radiographic Phenotype. Cancer Research. 2017;77:e104.

[28] Wallis TSA, Funke CM, Ecker AS, Gatys LA, Wichmann FA, Bethge M. A parametric texture model based on deep convolutional features closely matches texture appearance for humans. J Vis. 2017;17:5.

[29] National Comprehensive Cancer Network. NCCN guidelines https://www.nccn.org/professionals/physician_gls/f_guidelines.asp.

[30] Huang YQ, Liang CH, He L, Tian J, Liang CS, Chen X, et al. Development and Validation of a Radiomics Nomogram for Preoperative Prediction of Lymph Node Metastasis in Colorectal Cancer. Journal of clinical oncology : official journal of the American Society of Clinical Oncology. 2016;34:215764.

[31] Wang T, Gao T, Yang J, Yan X, Wang Y, Zhou X, et al. Preoperative prediction of pelvic lymph nodes metastasis in early-stage cervical cancer using radiomics nomogram developed based on T2-weighted MRI and diffusion-weighted imaging. European journal of radiology. 2019;114:128-35.

[32] Brembilla G, Dell'Oglio P, Stabile A, Ambrosi A, Cristel G, Brunetti L, et al. Preoperative multiparametric MRI of the prostate for the prediction of lymph node metastases in prostate cancer patients treated with extended pelvic lymph node dissection. European radiology. 2018;28:1969-76.

[33] He T, Puppala M, Ezeana CF, Huang YS, Chou PH, Yu X, et al. A Deep Learning-Based Decision Support Tool for Precision Risk Assessment of Breast Cancer. JCO clinical cancer informatics. 2019;3:112.

[34] Zheng X, Yao Z, Huang Y, Yu Y, Wang Y, Liu Y, et al. Deep learning radiomics can predict axillary lymph node status in early-stage breast cancer. Nature communications. 2020;11:1236.

[35] Mottet N, Bellmunt J, Bolla M, Briers E, Cumberbatch MG, De Santis M, et al. EAU-ESTRO-SIOG Guidelines on Prostate Cancer. Part 1: Screening, Diagnosis, and Local Treatment with Curative Intent. 
European urology. 2017;71:618-29.

[36] Gandaglia G, Ploussard G, Valerio M, Mattei A, Fiori C, Fossati N, et al. A Novel Nomogram to Identify Candidates for Extended Pelvic Lymph Node Dissection Among Patients with Clinically Localized Prostate Cancer Diagnosed with Magnetic Resonance Imaging-targeted and Systematic Biopsies. European urology. 2019;75:506-14.

[37] Gandaglia G, Martini A, Ploussard G, Fossati N, Stabile A, De Visschere P, et al. External Validation of the 2019 Briganti Nomogram for the Identification of Prostate Cancer Patients Who Should Be Considered for an Extended Pelvic Lymph Node Dissection. European urology. 2020.

[38] Gandaglia G, Fossati N, Zaffuto E, Bandini M, Dell'Oglio P, Bravi CA, et al. Development and Internal Validation of a Novel Model to Identify the Candidates for Extended Pelvic Lymph Node Dissection in Prostate Cancer. European urology. 2017;72:632-40.

\section{Figures}



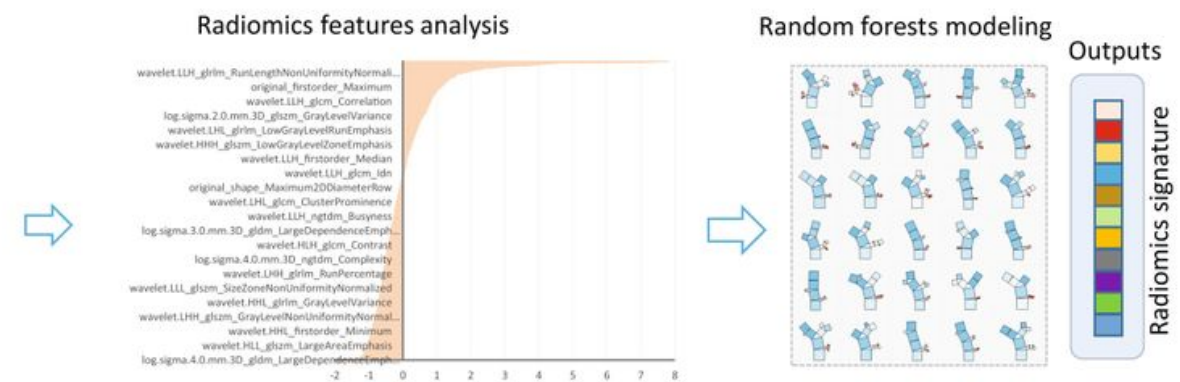

Step 2

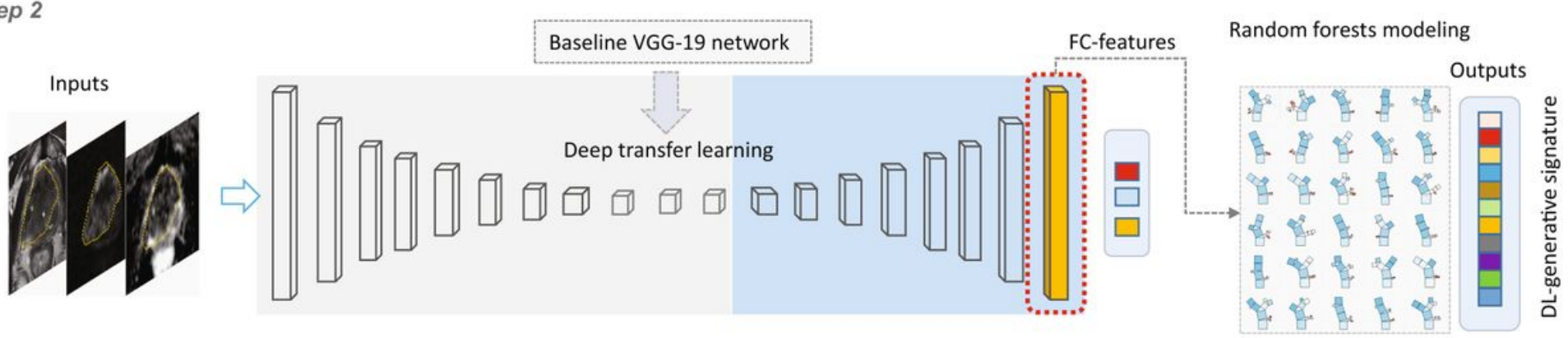

Step 3

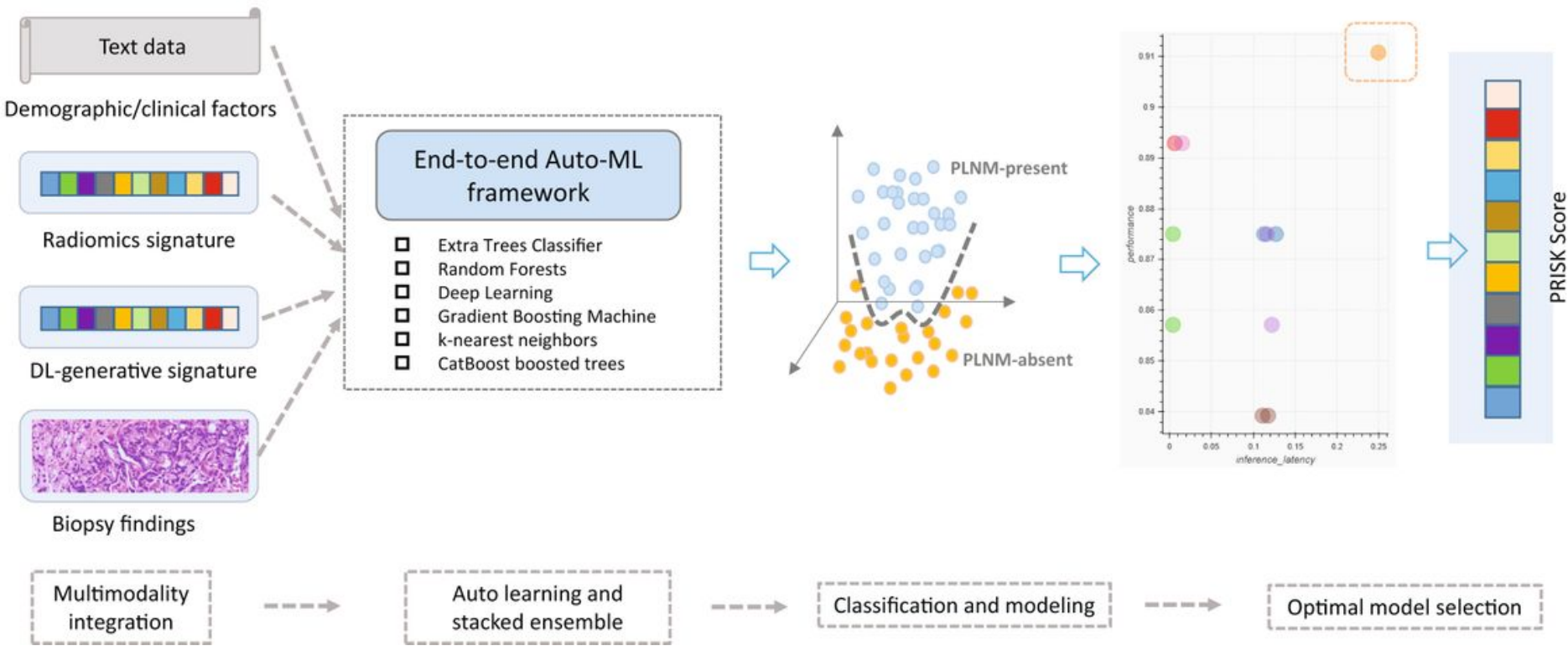

\section{Figure 1}

Flowcharts of imaging analysis and PRISK development. In step.1, radiomics features extracted from high-resolution T2-weighted imaging, high-b-value diffusion-weighted imaging and quantitative apparent diffusion coefficient images were ranked, selected and modeled by random forest classifier. In step 2 , deep-learning features extracted from the penultimate layer of the pre-trained VGG-19 model were ranked, selected and modeled by random forest classifier similarly. In step 3, three single-modality data including 18 clinico-radiological variables, outputs of radiomics machine learning and VGG-19 models (designated RML score and DL score) were integrated into the RRISK using an open-source auto Al-based analysis 
framework to derive a PLNM risk assessment. PRISK=Prostate Cancer Risk Calculator; RML = radiomics machine learning; $\mathrm{DL}$ = deep learning; PLNM=pelvic lymph node metastasis; $\mathrm{Al}=$ artificial intelligence.
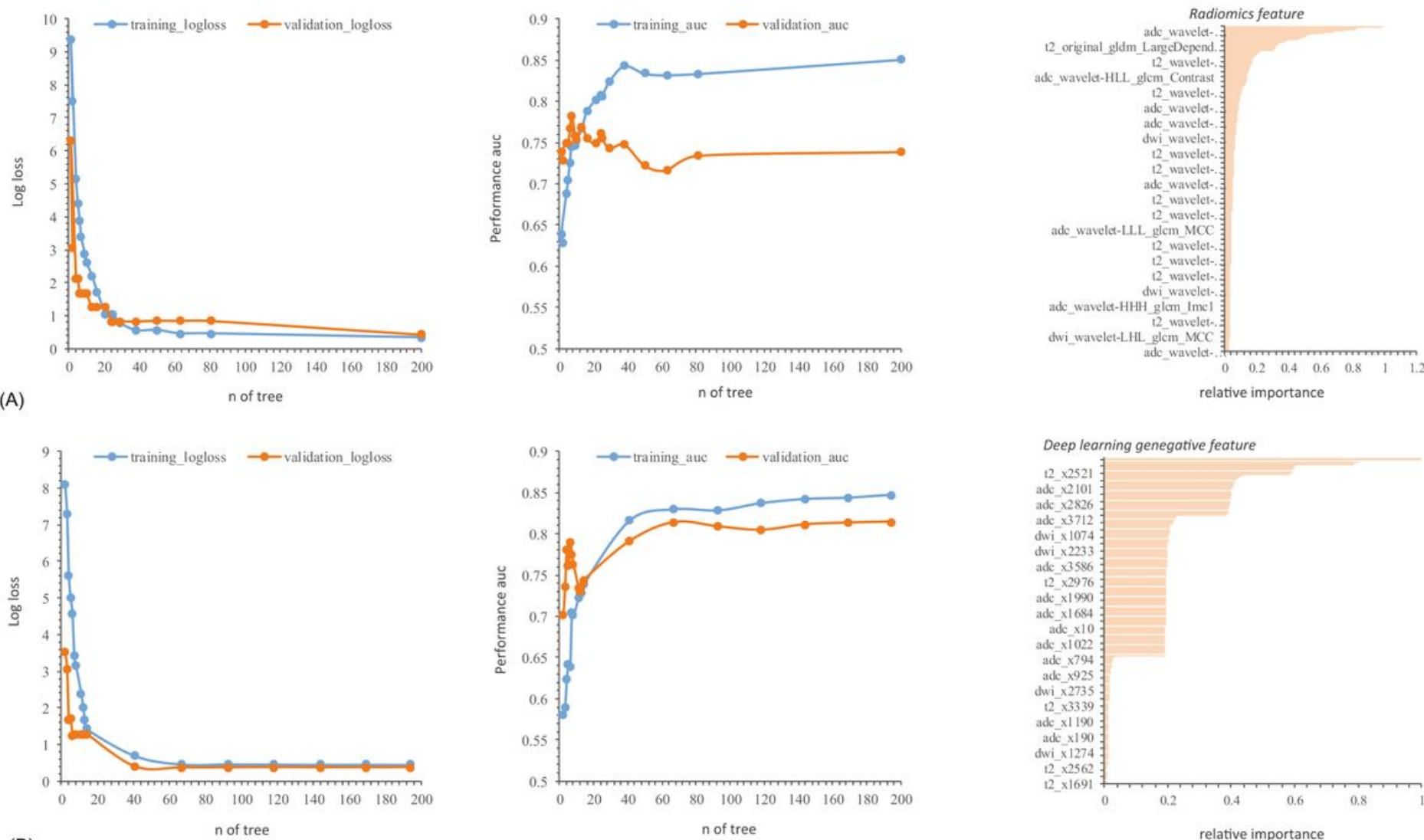

(B)
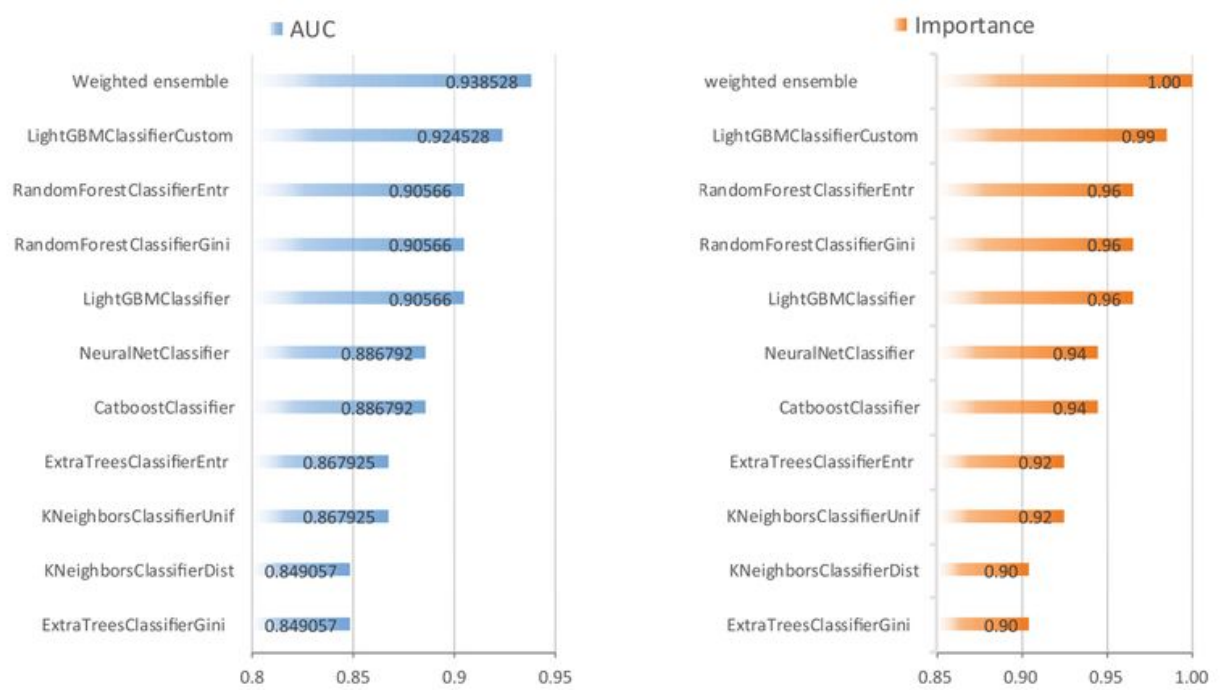

\section{Figure 2}

Graphs show results of (A.B) radiomics and deep learning features analysis with random forests and (C) ensemble of the PRISK model. (A.B) The detail log loss, performance and the importance of features ranked by mean decrease accuracy in radiomics machine learning and deep learning analysis. (C) The scaled importance of multimodality features and performance of auto-ensemble models using $\mathrm{Al}$ algorithms. 

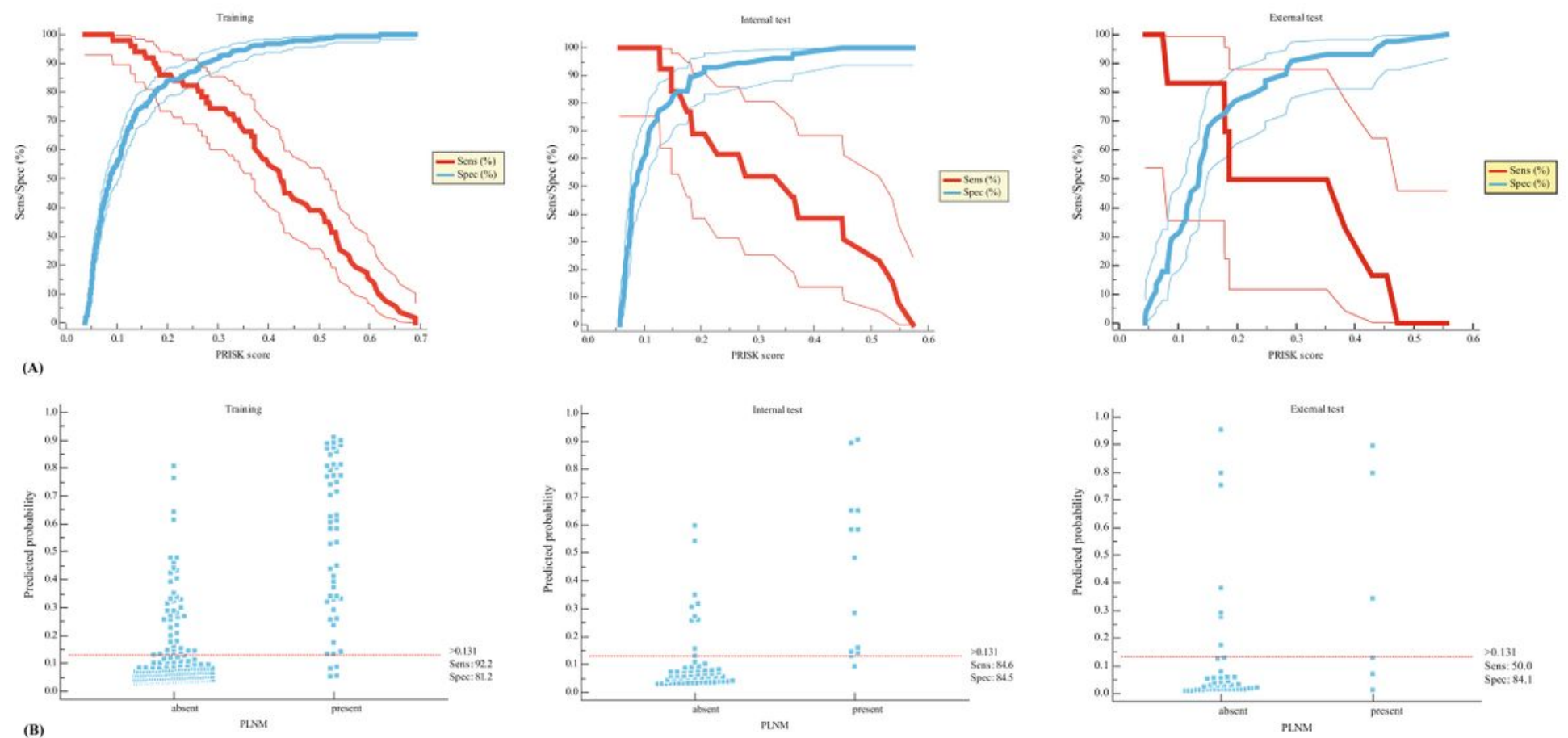

Diagnostic performance of predictive models for $\mathrm{N}$-staging

\begin{tabular}{|c|c|c|c|c|c|c|c|c|c|}
\hline \multirow{2}{*}{ Model } & \multicolumn{3}{|c|}{ Training/Validation $(n=280)$} & \multicolumn{3}{|c|}{ Internal Test $(n=71)$} & \multicolumn{3}{|c|}{ External Test $(n=50)$} \\
\hline & AUC & Sens & Spec & AUC & Sens & Spec & AUC & Sens & Spec \\
\hline RF scone & $0.859(0.812-0.897)$ & $44 / 51(0.863)$ & $167 / 229(0.730)$ & $0.829(0.721-0.908)$ & $11 / 13(0.846)$ & $32 / 58(0.552)$ & $0.784(0.645-0.888)$ & $4 / 6(0.667)$ & $32 / 44(0.727)$ \\
\hline VGG-19 scone & $0.856(0.809-0.895)$ & $36 / 51(0.706)$ & $200 / 229(0.873)$ & $0.841(0.735-0.917)$ & $6 / 13(0.462)$ & $51 / 58(0.880)$ & $0.777(0.637-0.882)$ & $3 / 6(0.500)$ & $38 / 44(0.864)$ \\
\hline Clinical & $0.902(0.861-0.934)$ & $43 / 51(0.843)$ & $192 / 229(0.838)$ & $0.875(0.775-0.942)$ & $10 / 13(0.769)$ & $48 / 58(0.828)$ & $0.648(0.500-0.777)$ & $4 / 6(0.667)$ & $29 / 44(0.659)$ \\
\hline Clinical $+R M L$ & $0.920(0.881-0.949)$ & $44 / 51(0.863)$ & $197 / 229(0.860)$ & $0.926(0.838-0.975)$ & $10 / 13(0.769)$ & $49 / 58(0.845)$ & $0.716(0.571-0.834)$ & $4 / 6(0.667$ & $32 / 44(0.727)$ \\
\hline Clinical $+D L$ & $0.916(0.877-0.946)$ & $44 / 51(0.863)$ & $195 / 229(0.852)$ & $0.910(0.818-0.965)$ & $10 / 13(0.769)$ & $50 / 58(0.862)$ & $0.712(0.567-0.831)$ & $4 / 6(0.667)$ & $31 / 44(0.705)$ \\
\hline PRISK & $0.932(0.895-0.958)$ & $47 / 51(0.922)$ & $186 / 229(0.812)$ & $0.924(0.837-0.974)$ & $11 / 13(0.846)$ & $49 / 58(0.845)$ & $0.758(0.616-0.868)$ & $3 / 6(0.500)$ & $37 / 44(0.841)$ \\
\hline
\end{tabular}

Note. MSKCC = Memorial Sloan Kettering Cancer Center (Ref. 7); Briganti score (Ref. 6); PRISK = Prostate Cancer Risk Calculator; RML = radiomics machine-learning; DL $=$ deep learning; AUC = area under the receiver operating characteristic curve; $\mathrm{ACC}=$ accuracy; Sens $=$ sensitivity; $\mathrm{Spec}=$ specificity.

(C)

\section{Figure 3}

Graphs show (A) the Sens and Spec curves, (B) the plots vs criterion values in classification of PLNM status using PRISK score and (C) diagnostic performance of predictive models for $\mathrm{N}$-staging. (A) The solid lines are created by sweeping a threshold over the predicted probability and the hidden lines represent the $95 \%$ confidence interval. (B) Based on the optimal threshold of 0.131 , the sensitivity is 0.992 vs 0.846 vs 0.500 and the specificity is 0.812 vs 0.845 vs 0.841 in in training/validation, internal test and external test cohorts, respectively. (C) Compared with other modalities/features integrations, PRISK performs best in the training/validation and internal test sets and performs comparably in the external test. Sens = sensitivity; Spec = specificity; PRISK=Prostate Cancer Risk Calculator; PLNM=pelvic lymph node metastasis; $\mathrm{RML}$ = radiomics machine learning; $\mathrm{DL}$ = deep learning; $\mathrm{AUC}=$ area under the receiver operating characteristic curve. 

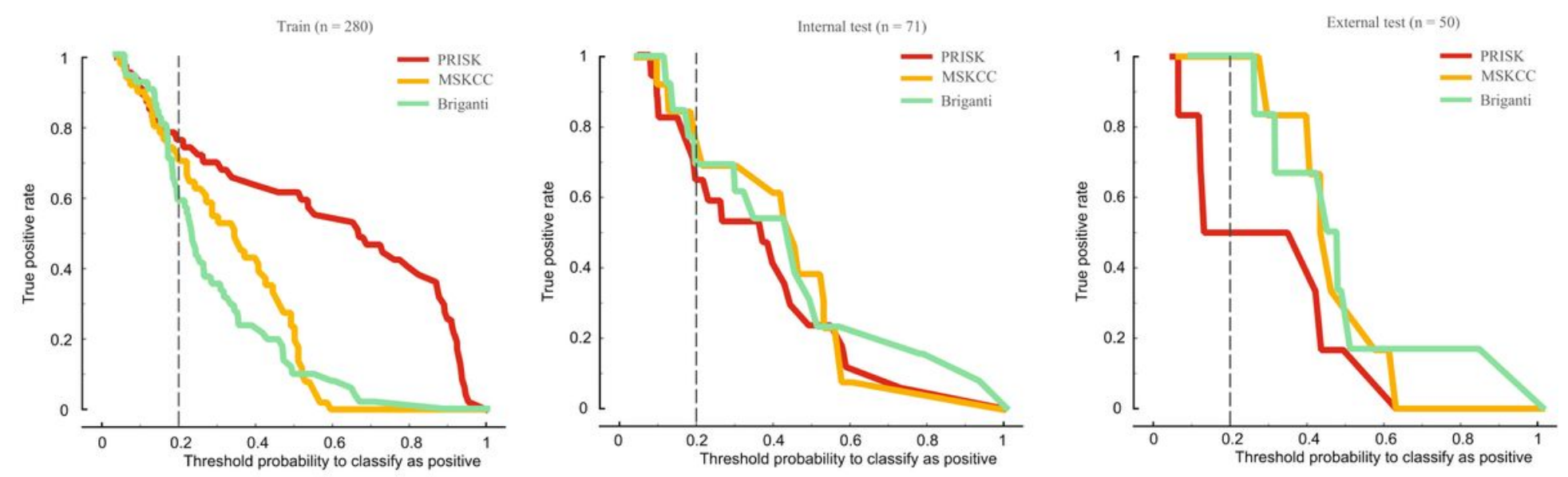

(1)
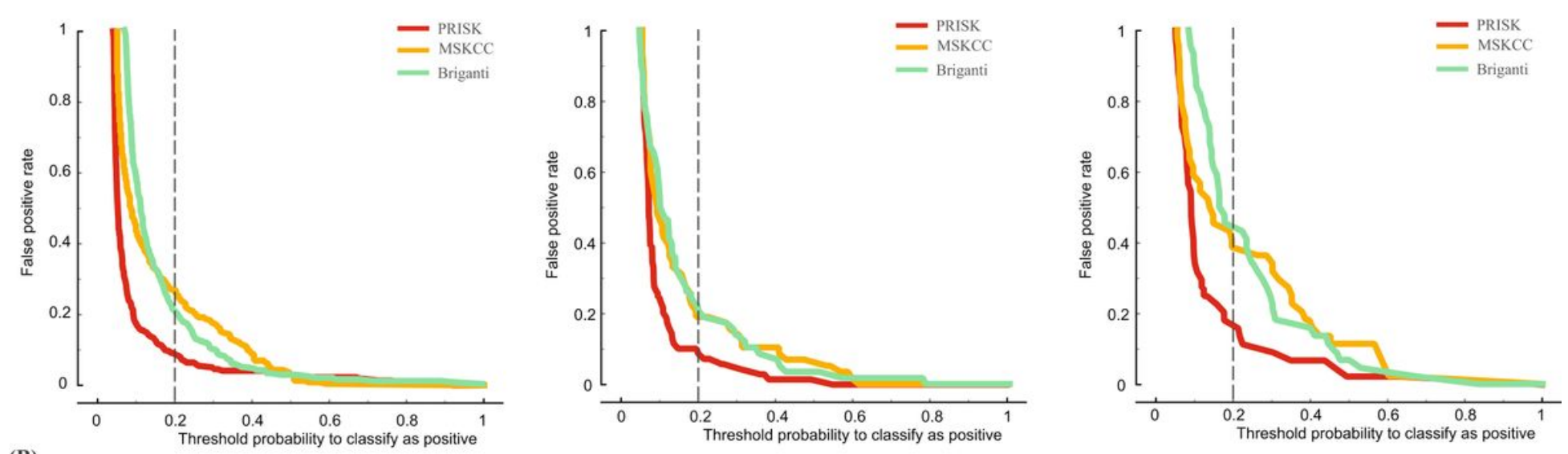

\section{Figure 4}

Graphs show (A) true positive rate and (B) false negative rate of MSKCC, Briganti and PRISK score for the prediction of PLNM in training/validation, internal test and external test cohorts respectively. For training/validation and internal test cohorts, PRISK resulted in similar true positive rate while achieved notably lower false positive rate at threshold probabilities of PLNM $<60 \%$ compared to MSKCC and Briganti score. For external test cohort, PRISK resulted in lower true positive rate and lower false positive rate compared to MSKCC and Briganti score. PRISK=Prostate Cancer Risk Calculator; PLNM=pelvic lymph node metastasis; MSKCC = Memorial Sloan Kettering Cancer Center. 

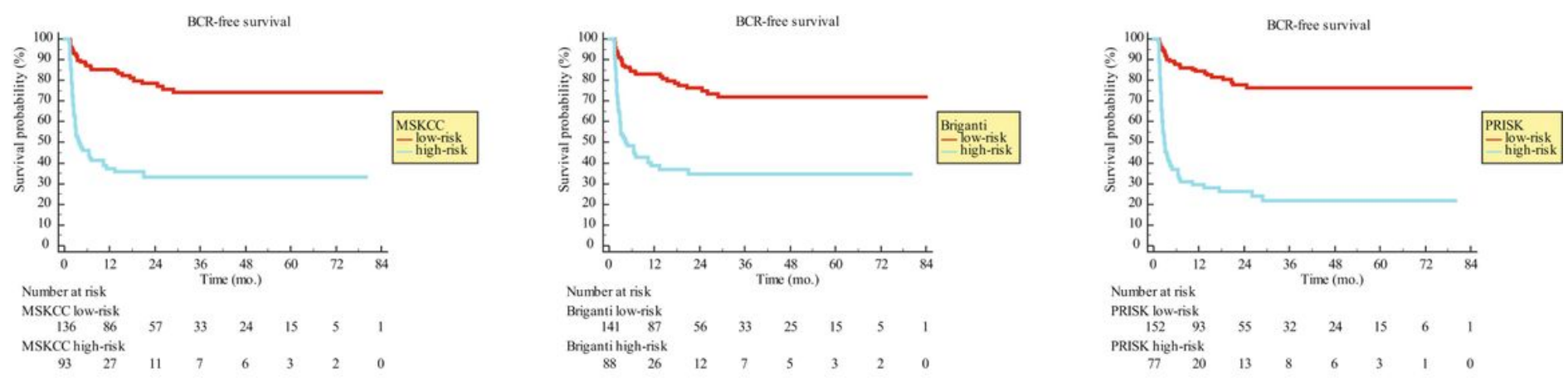

(A)
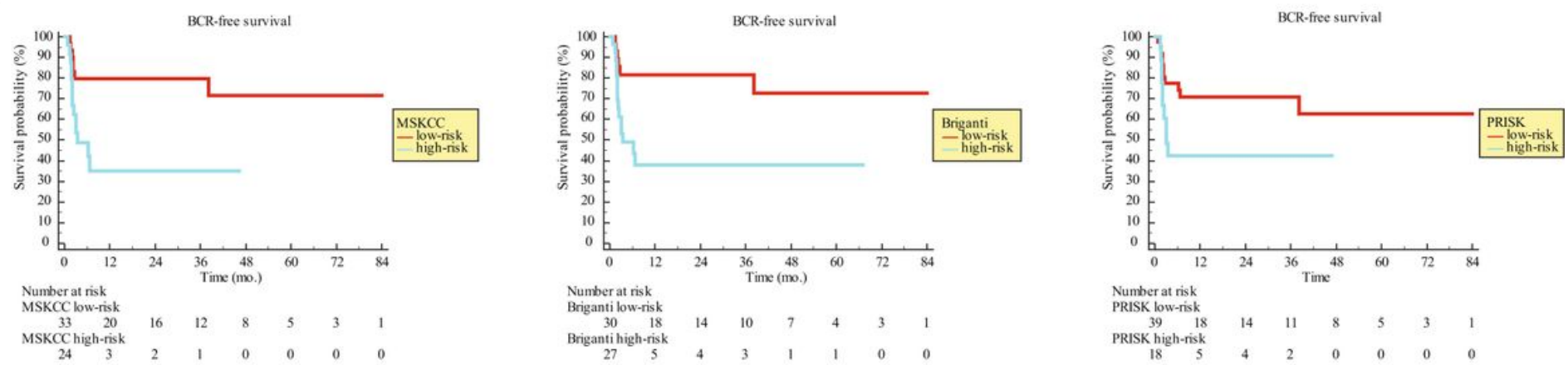

(B)
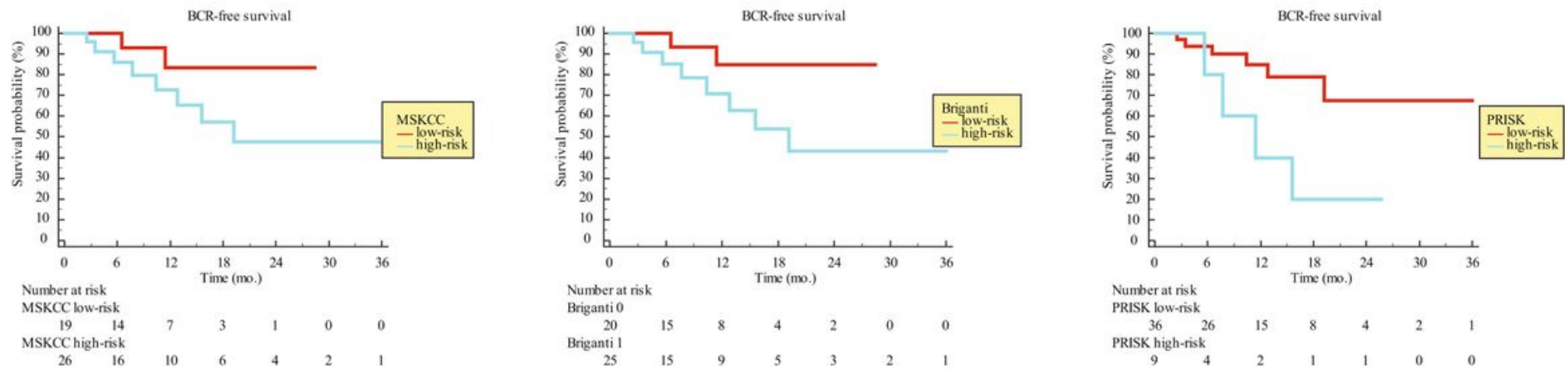

\section{Figure 5}

Kaplan-Meier survival curves of BCR according to the three prognostic models (MSKCC, Briganti and PRISK) in training/validation, internal test and external test cohorts respectively. The results reflect significantly different BCR-free survival in the subgroups stratified by MSKCC, Briganti and PRISK score, implying the prognostic relevance on short and long-term management of patients. PRISK=Prostate Cancer Risk Calculator; MSKCC = Memorial Sloan Kettering Cancer Center; BCR = biochemical recurrence .

\section{Supplementary Files}

This is a list of supplementary files associated with this preprint. Click to download.

- supplementaryData1.docx

- supplementaryData1.docx

- SupplementaryFig.1.eps

- SupplementaryFig.1.eps 
- SupplementaryTable1.doc

- SupplementaryTable1.doc 